\title{
BMJ Open Factors affecting Japanese retirees' healthcare service utilisation in Malaysia: a qualitative study
}

\author{
Ayako Kohno, ${ }^{1,2}$ Nik Daliana Nik Farid, ${ }^{1}$ Ghazali Musa, ${ }^{3}$ Norlaili Abdul Aziz, ${ }^{1}$ \\ Takeo Nakayama, ${ }^{2}$ Maznah Dahlui ${ }^{1,4}$
}

To cite: Kohno A, Nik Farid ND, Musa G, et al. Factors affecting Japanese retirees' healthcare service utilisation in Malaysia: a qualitative study. BMJ Open 2016;6:e010668.

doi:10.1136/bmjopen-2015010668

- Prepublication history for this paper is available online. To view these files please visit the journal online (http://dx.doi.org/10.1136/ bmjopen-2015-010668).

Received 26 November 2015 Revised 29 January 2016 Accepted 17 February 2016

CrossMark

\footnotetext{
${ }^{1}$ Department of Social and Preventive Medicine, Faculty of Medicine, University of Malaya, Kuala Lumpur, Malaysia

2Department of Health Informatics, Kyoto University School of Public Health, Kyoto, Japan

${ }^{3}$ Faculty of Business and Accountancy, University of Malaya, Kuala Lumpur, Malaysia

${ }^{4}$ Faculty of Medicine, Julius Centre, University of Malaya (JCUM), University of Malaya, Kuala Lumpur, Malaysia
}

Correspondence to Ayako Kohno;

kohnoayako@gmail.com

\section{ABSTRACT}

Objective: While living overseas in another culture, retirees need to adapt to a new environment but often this causes difficulties, particularly among those elderly who require healthcare services. This study examines factors affecting healthcare service utilisation among Japanese retirees in Malaysia.

Design: We conducted 6 focus group discussions with Japanese retirees and interviewed 8 relevant medical services providers in-depth. Guided by the Andersen Healthcare Utilisation Model, we managed and analysed the data, using QSR NVivo 10 software and the directed content analysis method.

Setting: We interviewed participants at Japan Clubs and their offices.

Participants: 30 Japanese retirees who live in Kuala Lumpur and Ipoh, and 8 medical services providers.

Results: We identified health beliefs, medical symptoms and health insurance as the 3 most important themes, respectively, representing the 3 dimensions within the Andersen Healthcare Utilisation Model. Additionally, language barriers, voluntary health repatriation to Japan and psychological support were unique themes that influence healthcare service utilisation among Japanese retirees.

Conclusions: The healthcare service utilisation among Japanese retirees in Malaysia could be partially explained by the Andersen Healthcare Utilisation Model, together with some factors that were unique findings to this study. Healthcare service utilisation among Japanese retirees in Malaysia could be improved by alleviating negative health beliefs through awareness programmes for Japanese retirees about the healthcare systems and cultural aspects of medical care in Malaysia.

\section{INTRODUCTION}

International retirement migration (IRM) is a growing phenomenon on a global scale. ${ }^{1-5}$ Retirement abroad is also popular among Japanese retirees. $^{6}$ For 9 consecutive years since 2006, Japanese retirees have ranked Malaysia as the most preferred country abroad to retire in. ${ }^{7}$ Malaysia My Second

\section{Strengths and limitations of this study}

- The study provides detailed insights of how Japanese retirees utilise healthcare service in Malaysia.

- It reveals factors that affect the healthcare service utilisation patterns among Japanese retirees in Malaysia.

- The research does not focus on specific ailments such as hypertension, diabetes, cardiovascular diseases and rheumatism, but, rather, centres on general health ailments experienced by Japanese retirees living in Malaysia.

- A qualitative study with recruited participants describes only a specific phenomenon; for better generalisability of the findings, a quantitative study that gains a representative sample of Japanese retirees in Malaysia is required to objectively measure the perceptions of crucial themes such as health beliefs, medical symptoms and health insurance of retirees living abroad.

Home (MM2H) was launched in 2002, which is a long-term residency programme to attract foreigners who meet certain criteriaproviding proof of financial assets and simple health records-to be granted a multiple-entry social visit pass visa for up to 10 years. $^{8} 9$ The Malaysian government invests considerable efforts to promote the country as a retirement destination for Japanese, by holding information seminars in various cities in Japan.

For Japanese retirees who utilise this programme, the primary purpose for choosing Malaysia is to enjoy living in a warm climate, to explore the cultural and natural attractions that the country offers, and to take advantage of the relatively inexpensive living cost. ${ }^{10}$ Although there are many benefits of retiring abroad, there are also some concerns. Elderly Japanese retirees who live abroad face some difficulties, especially in adapting to the different culture. However, perhaps the most 
important concern among elderly retirees in general, and not only to those from Japan, is healthcare service. At old age, many retirees have chronic ailments such as arthritis, diabetes, hernia and high blood pressure. ${ }^{11}$ To maintain their health and to seek treatment when needed, they visit clinics and hospitals in Malaysia.

In Malaysia, Japanese retirees may experience difficulty in accessing medical service because the healthcare system is different from what they are familiar with. Although a number of studies exist in Europe and the USA, there has been a paucity of academic research in areas of retirement abroad in Asia from the perspective of Asian retirees..$^{2-4}{ }^{12}$ In Malaysia, some studies have examined the motivation of Japanese retirees who live in Malaysia from a tourism perspective; however, research focusing on medical and healthcare issues among them does not exist. ${ }^{9} 1^{13-15}$ Therefore, the main objective of this study is to examine the factors that affect healthcare service utilisation among Japanese retirees in Malaysia. To gain a structured understanding of the issue, we adopted the Andersen Healthcare Utilisation Model (AHUM) as the conceptual framework for the research'. There are three factors in the AHUM that explain the individual's healthcare service utilisation, and these are 'predisposing characteristics', 'enabling resources' and 'need'. 'Predisposing characteristics' include variables such as age, gender, marital status and educational level. 'Enabling resources' are what support or impede a patient's access to healthcare. While 'need' is the perceived and evaluated requirements of the patients. This model was originally developed in the USA in the 1960s, first focusing on the family as a unit of analysis in healthcare service utilisation. It has been critically reviewed and adopted by other researchers, to become a more robust model, encompassing a wide variety of issues associated with healthcare service utilisation. ${ }^{16}$ The model has been applied to the research analyses of homeless people, ${ }^{17}$ equitable healthcare service for patients with cancer $^{18}$ and medication adherence in older adults. ${ }^{19}$ Figure 1 shows the simplified version of the AHUM.

\section{METHODS}

\section{Study design}

This qualitative study uses the directed content analysis method. ${ }^{20}$ We summarise the study findings using the AHUM as a conceptual framework, and extend the theory by adding new findings to the existing information. We conducted focus group discussions (FGDs) and semistructured interviews ${ }^{21}$ to examine the factors influencing healthcare service utilisation among Japanese retirees in Malaysia. This inductive approach allows the discovery of pertinent perspectives in healthcare service utilisation among them.

\section{Definitions}

Using the directed content analysis method, we prepared three categories of predetermined codes prior to analysis of the transcripts'. Table 1 shows the list of coding categories, their operational definitions and predetermined codes.

\section{Participants}

We conducted six FGDs ${ }^{22}$ with 30 Japanese retirees who reside in two cities in Malaysia: Kuala Lumpur and Ipoh. The participants were 16 males and 14 females, aged from 54 to 79 years (average age 65.2 years). Of the 30 participants, 16 retirees were couples. The majority of the participants (18 of the 30) had a university degree. The average stay in Malaysia was 5.5 years, with minimum stay half a year and maximum stay 20 years. The participants were recruited using the snowball sampling method. ${ }^{23}$ Those who agreed to take part in the discussions were requested to suggest other Japanese retirees whom they thought would be interested in taking part in the next discussion. Additionally, we used a members-only social network site (SNS) webpage (http://malaysia-navi.vivian.jp/sns/) for Japanese retirees who reside in Malaysia, to announce our FGD meetings, and recruitment was made by sending emails and messages to the retirees.

We also conducted in-depth interviews (IDIs) ${ }^{24}$ with eight people who provide services to Japanese retirees in Malaysia, among whom are government officials, travel agents, hospital managers and Japanese interpreters in private hospitals. They were purposively chosen and a request for an interview was sent via email, fax and regular mail.

\section{Data collection}

We developed a discussion guide for a FGD and an interview guide for an IDI, which explained the study objective, and guided the participants through the discussion and interview. The FGD and IDI guides were developed in English as well as Japanese, and the first author (AK) carried out both FGD and IDI. FGD guides were pilot tested against two Japanese retirees and minor corrections were made according to the feedback received from them. The FGDs were conducted at Japan Clubs in Kuala Lumpur and Ipoh, and in a shared condominium space where one of the participants lives. They were conducted during the period of January-March 2015, with each FGD lasting, on average, $2 \mathrm{~h}$. Participants were asked about their general experience with regard to their healthcare service utilisation in Malaysia. This included their common practice in healthcare utilisation, worries and concerns, decision-making and satisfaction. For IDIs, we conducted the interviews at the work places of the participants. All the interviews took place in a quiet room where privacy was assured. IDIs were conducted from February-July 2015, with an average duration of $1 \mathrm{~h}$ for each. Prior to conducting the interview, we explained the purpose of the study and gained the participants' consent for their involvement. We enquired about their perceptions of the Japanese retirees' characteristics, the MM2H programme and how 
Figure 1 Simplified version of behavioural model of health services use adopted from Andersen Healthcare Utilisation Model (AHUM).

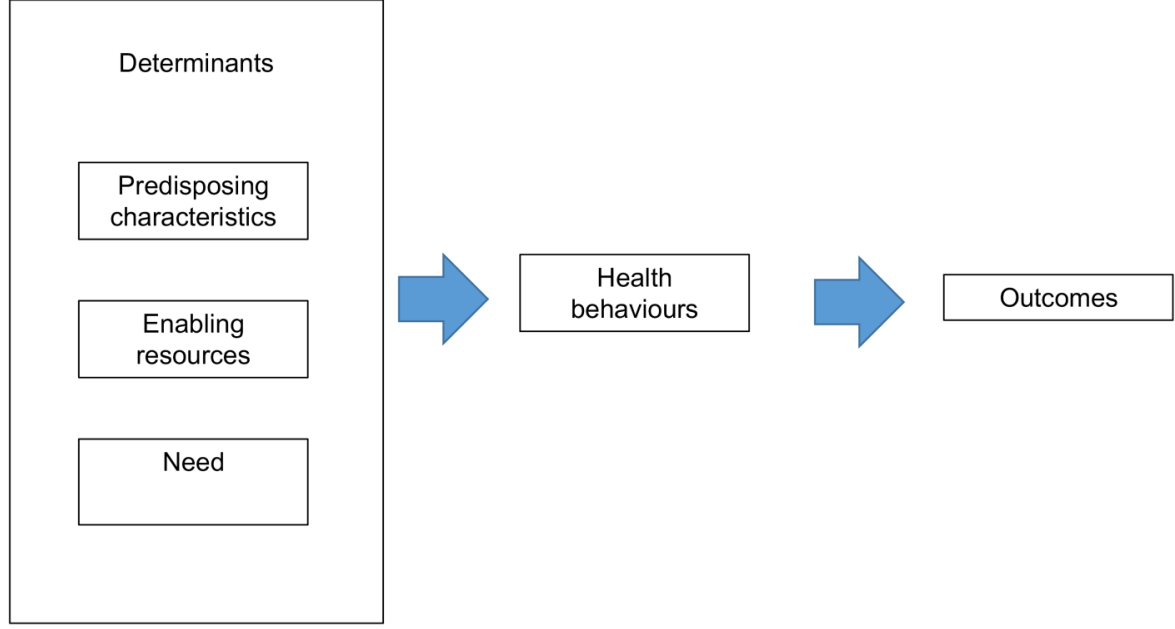

Japanese retirees utilise healthcare services in Malaysia. With permission from the participants, all FGDs and IDIs were audio recorded.

\section{Data analysis}

All FGDs were carried out in the Japanese language, while for IDIs, some were carried out in English and some in Japanese. The FGDs and those IDIs in Japanese were later translated into English. We managed the data using QSR NVivo 10 software. Data saturation from the FGDs and IDIs was reached after conducting five FGDs and seven IDIs. At that stage no new themes emerged even by adding additional data. ${ }^{25}$ The analysis method employed directed content analysis, guided by the three categories specified in the AHUM. ${ }^{26}$ This study followed the items in the consolidated criteria for the reporting qualitative research (COREQ) checklist. ${ }^{27}$ As depicted in table 1, healthcare service utilisation is grouped into three categories: predisposing characteristics, enabling resources and need. ${ }^{162628}$ The operational definitions of three coding categories were developed prior to analysis, by taking into consideration the AHUM's concepts. ${ }^{26}$ After the initial coding process, we checked the data fitness to predetermined codes by reviewing the coded transcripts against the definitions of the coding categories, as listed in table 1 . Then, based on the frequency of participant's mentioning the codes, the important themes were selected. When we discovered discrepancies between the predetermined codes and the findings, the differences were considered to be the gap that exists between theory and actual cases. The gaps were assigned new codes (refer to box 1).

\section{RESULTS}

For the purpose of this study, we chose only one theme from each category (construct) in the AHUM, and elaborated on it in detail. The three selected themes are health beliefs (representing 'predisposing characteristics'), health insurance (representing 'enabling resources') and medical symptoms (representing 'need'). We additionally discovered another three themes with the highest frequency in terms of participant numbers that are uniquely important to the retirees, but not within the AHUM codes. These were language barriers, voluntary health repatriation to Japan and psychological support.

\section{Health beliefs}

Within the coding category of predisposing characteristics, a total of $80 \%$ of the FGD participants and $88 \%$ of IDI participants mentioned health beliefs. According to Andersen, health beliefs include a wide range of personal thinking and behaviours such as attitudes, values and knowledge that people develop throughout their lives, pertaining to healthcare services. Health beliefs influence the way people formulate ideas about the need for healthcare services. ${ }^{28}$ For Japanese retirees in Malaysia, the beliefs had been established beforehand, through their previous encounters with healthcare services in Japan. Any negative discrepancy between the medical systems in Japan and Malaysia would be perceived as unfamiliar/unacceptable medical services. We recorded a specific element commonly rooted in the perception of Japanese retirees in Malaysia. They placed equal emphasis of both the actual medical treatment/ procedure and the mental care needed by them from healthcare providers, to provide support and alleviate their anxieties when going through the medical treatment/procedures. For this reason, those physicians who take time with their patients to explain processes in detail and who provide immaculate management and handling of patients were preferred by the Japanese retirees. Here, Japanese interpreters in private hospitals play a crucial role to ease their anxieties while the Japanese retiree patients receive medical treatment/ undergo procedures in Malaysia.

Basically, I adopt (the idea of) preventive medicine, I try various things to not become sick, so, about the worries and concerns, I do not have. Up until now, I've been having medical check-ups once a year in Japan, and now 
Table 1 List of coding categories, operational definitions and predetermined codes

\begin{tabular}{|c|c|c|}
\hline Coding categories & Operational definitions & Predetermined codes \\
\hline $\begin{array}{l}\text { Predisposing } \\
\text { characteristics }\end{array}$ & $\begin{array}{l}\text { Any variable that is considered a predisposition of an individual. } \\
\text { Demographic characteristics such as age, gender, marital status and } \\
\text { education level, occupation and ethnicity, are the examples of } \\
\text { predisposing characteristics. Also, health beliefs are considered to be } \\
\text { one of the predisposing characteristics, such as attitudes, values and } \\
\text { knowledge about healthcare. In addition, a broader concept of } \\
\text { predisposing characteristics encompasses social networks and } \\
\text { interactions that individuals have. }\end{array}$ & $\begin{array}{l}\text { Chronic symptom history } \\
\text { Health beliefs } \\
\text { - Personal health practices } \\
\text { (diet, exercise, etc) } \\
\text { Preventive health } \\
\text { behaviours } \\
\text { Social network and } \\
\text { interaction } \\
\text { - Values and cultural norms }\end{array}$ \\
\hline Enabling resources & $\begin{array}{l}\text { Any variables that support or impede a patient's access to healthcare } \\
\text { are generally considered as enabling resources. Financial factors } \\
\text { such as economic status and health insurance determine healthcare } \\
\text { service utilisation. Lack of the enabling resources may delay the } \\
\text { healthcare service utilisation. }\end{array}$ & $\begin{array}{l}\text { - Distance to hospitals } \\
\text { Health insurance } \\
\text { - Transport to hospitals } \\
\text { - Waiting time }\end{array}$ \\
\hline Need & $\begin{array}{l}\text { Any variables for need consist of perceived and evaluated need. } \\
\text { Perceived need is the need created based on how people judge their } \\
\text { general health status. Emotional response to pain and illness are } \\
\text { also included in this category. Evaluated need is the judgement } \\
\text { made by medical professionals regarding the need for healthcare } \\
\text { provision to the patients. }\end{array}$ & $\begin{array}{l}\text { Perceived need } \\
\text { Medical symptoms } \\
\text { Satisfaction of received } \\
\text { healthcare } \\
\text { Self-rated general health } \\
\text { status } \\
\text { Worry about their health } \\
\text { condition }\end{array}$ \\
\hline
\end{tabular}

I am looking for a medical institution in Malaysia which can offer me a similar level of service. (Male Japanese retiree, FGD1)

The systems, the doctors, they operate within the hospitals, are individually owned, and the medical staff belong to the hospitals, so, between the doctors and the staff, the relationship, I thought it is not the same (as the case in Japanese hospital), so the nurses won't tell the doctors immediately, I suspect that that kind of thing exist. (Female Japanese retiree, FGD2)

Well, the physicians, they skip their explanations sometimes. Because they don't have time. So, the patients, even though they hear about the name of their diagnosed ailments, even in Japanese language, sometimes it is difficult to understand. So, I explain that to the patient in Japanese language, but they say "what is it?" There are many cases. Then, I try to break it down to easy words

\section{Box 1 List of newly added codes}

\section{Codes}

- Language barrier

- Voluntary health repatriation to Japan

- Psychological support

- Having multiple appointments in one visit

- Healthcare information from other Japanese retirees

- Outreach and educational activities by hospitals

- Second opinion

- Advanced medical services in Malaysia

Change in lifestyle patterns and add an explanation. Otherwise, the patient cannot understand. (Interpreter, IDI7)

Some people have preconceived ideas about their health ailments, and even though the diagnoses are made by the doctors, these people are unable to instantly change their mindset, so they are not convinced about the diagnoses. In such cases, the role of the healthcare provider is to provide the correct name of the ailment, as well as to correct all the inaccurate self-health assessments that the patients had prior to visiting the hospital.

Well, perhaps, the disease for internal organs, when they experience the pain in the left shoulder, and they suspect the heart disease. They saw the information on the Internet, and definitely, that is what they have, they believe strongly so, and they go to the hospital, there are many who are like that. So, for those people, we need to show the objective data, in that case, we need to show the blood test results, and the diagnostic imaging results, and based on these information, we explain to them, and overall, that is not the problem, and there is no problem with the blood test, we explain, and after all, it must be the muscle pain, we explain to them. (Interpreter, IDI7)

Well, the shockwave I received (in Malaysia for ureteral calculi) it was hurting me a lot after I received treatment. In my family, as most of my brothers have the same symptom, they say it was not like that, it did not hurt. I went back to a hospital in Japan, as I thought, well, the power (of the shockwave), it was too strong. So, in the case of Japan, they prefer safety and reassurance first, so (the power is) small, and hit it three or four times, but in 
here (Malaysia), in one time, they take away big time. I was bleeding badly, I think. The doctors in Japan said my kidney was swollen. (Female Japanese retiree, FGD2)

\section{Health insurance}

Within the coding category of enabling resources, a total of $67 \%$ participants and $88 \%$ of IDI participants mentioned health insurance. The AHUM considers insurance as an incentive mechanism to increase healthcare usage. $^{26}$ In this study, we found that most Japanese retirees preferred to use a cashless service (ie, health insurance) that they subscribe to. Using the service, the transaction is dealt with directly by hospitals and insurance companies. Therefore, for some retirees, healthcare insurance coverage is the key determinant in selecting a hospital.

Then, I went to private hospital next, in that case, I used the medical insurance, but that takes a lot of time... we have to write a lot, but, that is cashless, so it is very helpful. As I was having ureteral calculi, that shockwave and the hospitalization for 1 or 2 days, cost about 400,000 yen. So, the insurance is invaluable. (Female Japanese retiree, FGD2)

\section{Medical symptoms}

Within the coding category of need, $67 \%$ of the FGD participants and $50 \%$ of IDI participants mentioned medical symptoms. Perceived need is a crucial factor in the AHUM. Andersen and Davidson ${ }^{28}$ explained that perceived need is largely a social phenomenon, stemming from the proactive desire to seek out healthcare services. Health symptoms experienced by Japanese retirees in Malaysia vary greatly, and include those of dermatology, gynaecology, autoimmunity, otolaryngology, ophthalmology, orthopaedic or cardiovascular or psychiatric conditions and of gastroenterology. Also, there are common types of chronic illnesses such as diabetes and hypertension. There are frequent cases of infectious diseases such as dengue, influenza and food poisoning. Basically, these medical symptoms can be categorised as acute or non-acute. It should be highlighted that acute symptoms are more common among Japanese retirees in Malaysia, who often embark on an active lifestyle. Those who appreciate the outdoors and play sports commonly experience unexpected injuries and pain, as well as contracting infectious diseases. Ageing and having a weakened immune system may exacerbate these symptoms.

For the diabetes, usually I go to internal medicine department and the ophthalmology department. So, these two points, it will all be the follow-ups. The internal medicine, well, the measured value for diabetes are the blood sugar level and A1C level. And in ophthalmology department, first of all, they observe the ocular fundus. Those are the regular checkups that I receive. And, in addition, I also do my visual field test. As I may have higher chances of getting glaucoma, now, I try to prevent and receive the checkup. (Male Japanese retiree, FGD3)

Every day, because there are a lot of sunshine, the exhaustion of physical strength, that is probably the reason. We have many patients with shingles. (Interpreter, IDI7)

We also identified three themes that are not described by the AHUM, but that are important in their influence on healthcare service utilisation among Japanese retirees in Malaysia. These are language barriers, health repatriation to Japan and psychological support.

\section{Language barriers}

A total of $80 \%$ of FGD participants and $100 \%$ of IDI participants mentioned a language barrier. Communicating with physicians and nurses in English is difficult for most Japanese retirees, especially when they first visit the hospitals. With the availability of Japanese interpreters in some private hospitals, retirees can be informed of their symptoms and understand the diagnosis in Japanese. However, the medical systems and healthcare services in Malaysia differ from those in Japan. When patients visit a hospital for the first time, they need to receive orientation guidance in order to go over the anticipated medical treatment/procedures with the Japanese interpreters. Many Japanese retirees as well as Japanese interpreters commented on the expected service when a new patient visits the hospital for the first time.

And then, they took me to the emergency, and they examined me, and did the testing, and various things, but well, because of the language problem, and there were no explanations, I was not sure what they did to me, and the injection, what was in it, I didn't understand, so for that reason, I was very anxious. (Female Japanese retiree, FGD2)

So, not only about diagnosis, but as there are some patients who cannot speak English at all, the way of receiving diagnosis, and how to deal with the reception, and depending on the diagnosis, there are tests afterwards, such as X-rays and respiratory test, so how to go through these? Then, at the end, to receive medication at the pharmacy, and how to make the payment, and how to apply for the insurance claim, those, all of them, I help with. (Interpreter, IDI6)

\section{Health repatriation to Japan}

A total of $60 \%$ of FGD participants and $100 \%$ of IDI participants mentioned voluntary health repatriation to Japan. It is surprising but an understandable fact that many Japanese retirees go back to Japan when they have a serious illnesses. From the standpoint of the destination country, this phenomenon is a missed opportunity for the healthcare industry. There are several reasons why these retirees prefer to return to Japan for treatment. For example, when surgery is required, it is a big 
life event, so the Japanese retirees prefer to choose a hospital in their home country, to eliminate worries of miscommunication in a foreign country and culture. Also, when family support is direly needed, it is most desirable to be near to relatives and family members. The Japanese retirees expressed their intention to return to Japan if some serious health deterioration were to occur.

And, if there is something big to our health happened, it is probably better that we return (to Japan). If nothing happened, and everything goes well, we want to stay longer. I feel like that now. (Male Japanese retiree, FGD1)

For now, I am thinking, I should go back to Japan and receive medical treatment. That is my honest feeling. (Female Japanese retiree, FGD3)

\section{Psychological support}

A total of $50 \%$ of IDI participants and 7\% of FGD participants mentioned receiving psychological support and treatment. Some Japanese retirees in Malaysia experience symptoms of insomnia, depression and adjustment disorders that warrant psychological or psychiatric care. These retirees may need a medical prescription or counselling; however, the language barrier limits their access to such healthcare, even with the help of Japanese interpreters. Some developed the symptoms prior to arriving in Malaysia, while others experienced them while living in the country. For some retirees living in Malaysia as a couple, it is often the case that only one of them is more enthusiastic about living abroad, while the other does not necessarily look forward to making such a change. Other factors such as the character of the individual, the drastic change in the living environment and communication issues, are intertwined, causing some Japanese retirees in Malaysia to experience psychological disturbances.

Well, the doctor says "Well, let us relax a little bit more". Because you are coming here (in Malaysia) to enjoy. But, they cannot relax. Their character does not allow it, as they have been like that all these while, so it would be difficult to change their way, and because the surrounding environment is not that way, they feel the stress, I think. (Interpreter, IDI7)

The psychiatric department is difficult. The counselling, well, even the doctors in Japan, some of them do it thoroughly, and the others, they won't even listen to you and just prescribe the medication. Well, as I am not good at English, well, the counselling, I do not expect that much here (in Malaysia). (Female Japanese retiree, FGD5).

\section{DISCUSSIONS AND CONCLUSIONS}

The themes of health insurance, medical symptoms and psychological support will be omitted from the discussion as the management is largely carried out by medical providers (eg, doctors and insurance company).
In this section, we prioritise the themes that illustrate the internal aspects of Japanese retirees, and the conditions that can be managed by awareness programmes on medical services and culture in Malaysia. These themes are health repatriation to Japan, health beliefs and language barriers.

The emotions rooted behind the decision for voluntary health repatriation to Japan can be reasoned out. To understand their justification for such behaviour, we need to focus on their emotional transition. When one's health condition deteriorates, or when one is diagnosed with a serious ailment, the mind is preoccupied with a pessimistic outlook. Makowski et $a l^{29}$ commented that the elderly are more likely to avoid potentially negative situations by using their prior knowledge and wisdom. They implement situation selection strategies and choose what they consider to be the best solution to the given situation. For most Japanese retirees in Malaysia, going back to Japan (their homeland) to receive medical treatment is the safest and most feasible option. This reality poses challenges to healthcare providers and policy makers in Malaysia. To provide medical services to Japanese retirees in this country, healthcare providers not only have to deliver high quality healthcare, they must also ensure a satisfactory explanation and emotional reassurance. A study on the causes of suffering among patients in emergency rooms revealed a significant contribution of both physical pain and emotional distress. ${ }^{30}$ This observation was replicated in this study among Japanese retirees in Malaysia, with some highlighting the importance of both physical and psychological care. Healthcare providers in Malaysia need to be mindful of these holistic needs, which should be for Japanese retirees, as well as for all patients in general.

Andersen argued that, in order to bring changes to healthcare service utilisation, the variable must be changeable, or 'mutable', so that the status of influence to the patients can be modified with intervention. ${ }^{26}$ For this reason, demographic variables, such as age and gender, are not suitable variables for focus of intervention, as these cannot be altered. Health beliefs, ${ }^{26}$ on the other hand, can be modified, when new and appropriate information is provided to the patients, to educate and provide better awareness of the Malaysian medical system. The health beliefs among Japanese retirees in this country are characterised by narrow-mindedness, sometimes even prejudice, largely preconditioned by their satisfactory past experiences with the healthcare services in Japan. Based on this positive experience, they stereotype what healthcare services should be. We propose that an awareness programme be carried out, either by private hospitals or by the Malaysian government, to condition Japanese retirees to embrace and adapt to the Malaysian ways in healthcare service provision.

Language barriers are not merely the inability to comprehend a language, but also a lack of capacity to communicate with the physicians and nurses when diagnosed, with pharmacists when receiving medication 
and with receptionists when making payments. Therefore, various types of miscommunication are prone to occur among Japanese retirees in Malaysia. Furthermore, to decipher what is said and not said by the healthcare providers is confusing. Some Japanese retirees also make their own diagnoses, based largely on internet research or inferences drawn from their previous experiences with healthcare providers in Japan. The assistance of Japanese interpreters enhances their capacity to comprehend the language, but the communication aspect of language barriers is harder to overcome, even with the help of the interpreters. A sense of autonomy is missing, as communication efforts made by the patient go through the mind filter of the interpreter. Communication is not just about exchanging words, but also about sharing emotions. Establishing personal relations with harmonious interdependence is valued highly in some Asian cultures. ${ }^{31}$ In the Japanese culture, harmonisation is most crucial and important for satisfactory healthcare delivery and encounters. Facial expressions and eye contact are also important elements in communication. Non-verbal communication strategies can fill the voids created by lexical differences within the Japanese and English languages. ${ }^{32}$ Therefore, it is important that physicians, if even with a few words, talk directly with Japanese retiree patients, without the help of interpreters. Such outreach must be offered by physicians, nurses and hospital staff, to assure foreign patients that smooth communication is possible even without understanding all the words.

The strength of this study is its provision of in-depth insights into how Japanese retirees utilise healthcare services in Malaysia. In addition, this study reveals the factors affecting healthcare service utilisation patterns of Japanese retirees in Malaysia. A limitation is the lack of focus on specific ailments. By narrowing the research focus to specific ailments, such as hypertension, diabetes and depression, for example, the healthcare service utilisation pattern could be tailored more precisely for medical treatment, support and procedures. However, this study aimed to grasp the overall trends of healthcare service utilisation among Japanese retirees in Malaysia, to establish general baseline issues, evidenced from multiple perspectives and stakeholders. Other studies about healthcare service utilisation of retirees highlighted such topics as physicians' expression of personal interest in patients, length of time taken for an appointment and availability of physicians when needed. ${ }^{5}$ These were the views expressed by retirees in Western countries, and may differ from those of the Japanese retiree population. Another limitation is the choice of the method-a qualitative study-the results of which only represent the reality of the participants on a specific phenomenon. A quantitative study can further measure and quantify factors that affect healthcare service utilisation among Japanese retirees in Malaysia, the results often allow generalisation of the findings. In future, we plan to carry out quantitative research to quantify and verify the issues that we found in this study.
In conclusion, this study provides detailed and in-depth insight pertaining to healthcare service utilisation among Japanese retirees in Malaysia. We discovered that social issues such as language barriers and emotional reassurance closely influence the experience in healthcare service utilisation among Japanese retirees in this country. Hospital managers and policymakers need to be mindful of the importance of these two elements, to be incorporated in the planning and instituting of superior healthcare services and delivery especially to Japanese retirees. Future study may examine the satisfaction with healthcare services in Malaysia, and perhaps explore aspects of healthcare service utilisation for specific medical ailments (eg, hypertension, diabetes and depression). Medical services in Malaysia need to make a continuous effort to examine the ever changing and varied needs of MM2H participants to further attract a greater number of inbound retirees, and ensure the sustainability of this programme. Other than benefiting foreign retirees, improved healthcare services will eventually benefit the general population in Malaysia.

Acknowledgements The authors thank all those who participated in this study.

Contributors AK conceived the study design, and carried out the interviews and discussions. AK, NDNF, GM and NAA performed qualitative analyses. AK drafted the manuscript, which was edited by NDNF and GM. AK, NDNF, GM, NAA and MD contributed to project management and revision of the manuscript. All the authors read and approved the final manuscript.

Funding This work was supported by University of Malaya Research (grant number AADUN RP-026-2012C).

Disclaimer The funder had no role in study design, data collection and analysis, and decision to publish, nor in preparation of the manuscript.

Competing interests None declared.

Patient consent Obtained.

Ethics approval This study was approved by the Medical Ethics Committee of University of Malaya, Malaysia. (MECID number 201411-815).

Provenance and peer review Not commissioned; externally peer reviewed.

Data sharing statement No additional data are available.

Open Access This is an Open Access article distributed in accordance with the Creative Commons Attribution Non Commercial (CC BY-NC 4.0) license, which permits others to distribute, remix, adapt, build upon this work noncommercially, and license their derivative works on different terms, provided the original work is properly cited and the use is non-commercial. See: http:// creativecommons.org/licenses/by-nc/4.0/

\section{REFERENCES}

1. Warnes TAM. International retirement migration. International handbook of population aging. Vol 1. Springer, 2009:341-63.

2. Rodríguez V, Fernández-Mayoralas G, Rojo F. International retirement migration: retired Europeans living on the Costa del Sol, Spain. Popul Rev 2004;43:1-36.

3. Amin I, Ingman SR. Retiring in a foreign land: how do the American retirees deal with health care issues in Mexico? J Aging Emerg Economies 2010;2:22-35. https://www2.kent.edu/sociology/ resources/jaee/upload/retiring-in-a-foreign-land.pdf

4. Legido-Quigley $\mathrm{H}$, Nolte E, Green J, et al. The health care experiences of British pensioners migrating to Spain: a qualitative study. Health Policy 2012;105:46-54.

5. Sloane PD, Cohen LW, Haac BE, et al. Health care experiences of U.S. retirees living in Mexico and Panama: a qualitative study. BMC Health Serv Res 2013;13:411. 
6. Ono M. Long-Stay Tourism and International Retirement Migration: Japanese Retirees in Malaysia. Transnational Migration in East Asia Senri Ethnological Reports 2008;77:151-62. http://camel.minpaku. ac.jp/dspace/bitstream/10502/2043/1/SER77 013.pdf

7. Long Stay Foundation. Longstay Annual Statistical Report [Long Stay Chousa Toukei]. 2014. http://www.longstay.or.jp/product/booklist/

8. Ministry of Tourism and Culture Malaysia My Second Home Centre. MM2H Programme Statistics. [Internet Website]. In press. 2015.

9. Abdul-Aziz AR, Loh CL, Jaafar M. Malaysia's My Second Home (MM2H) Programme: an examination of Malaysia as a destination for international retirees. Tourism Manage 2014;40:203-12.

10. Miyazaki K. An aging society and migration to Asia and Oceania Transnational Migration in East Asia 2008:139-49. http://prami.ibero. $\mathrm{mx} / \mathrm{wp}$-content/uploads/2012/03/Demografia-y-migracion-enAsia-y-Oceania.pdf

11. Wong KM, Musa G. Retirement motivation among 'Malaysia My Second Home' participants. Tourism Manage 2014;40:141-54.

12. Breuer T. Retirement migration or rather second-home tourism? German Senior Citizens on the Canary Islands. Dedicated to Jürgen Bähr on his 65th birthday. Die Erde Z Ges Für 2005;136:313-33. http://epub.uni-regensburg.de/1114/1/JPG-313-333_neu.pdf

13. Wong KM, Musa G. Challenges of international retirees in second home destination: A phenomenological analysis. Tourism Manage Perspect 2015;15:81-90.

14. Wong KM, Musa G. International second home retirement motives in Malaysia: comparing British and Japanese retirees. Asia Pac J Tourism Res 2014:1-22. http://www.tandfonline.com/doi/abs/10. 1080/10941665.2014.950303\#.VbdCgfntmko

15. Stapa SH, Musaev T, Hieda N, et al. Issues of language choice, ethics and equity: Japanese retirees living in Malaysia as their second home. Lang Intercultural Commun 2013;13:60-77.

16. Andersen RM. National health surveys and the behavioral model of health services use. Med Care 2008;46:647-53.

17. Gelberg L, Andersen RM, Leake BD. The behavioral model for vulnerable populations: application to medical care use and outcomes for homeless people. Health Serv Res. 2000;34:1273.

18. Mandelblatt JS, Yabroff KR, Kerner JF. Equitable access to cancer services. Cancer 1999;86:2378-90.
19. Murray MD, Morrow DG, Weiner M, et al. A conceptual framework to study medication adherence in older adults. Am J Geriatr Pharmacother 2004;2:36-43.

20. Hsieh HF, Shannon SE. Three approaches to qualitative content analysis. Qual Health Res 2005;15:1277-88.

21. Liamputtong P. Research methods in health: foundations for evidence-based practice. Oxford University Press, 2013.

22. Wong LP. Focus group discussion: a tool for health and medical research. Singapore Med J 2008;49:256-60.

23. Biernacki P, Waldorf D. Snowball sampling: problems and techniques of chain referral sampling. Sociol Methods Res 1981:10:141-63.

24. Boyce C, Neale P. Conducting in-depth interviews: a guide for designing and conducting in-depth interviews for evaluation input. Watertown, MA: Pathfinder International, 2006.

25. Fusch PI, Ness LR. Are we there yet? Data saturation in qualitative research. Qual Rep 2015;20:1408-16.

26. Andersen RM. Revisiting the behavioral model and access to medical care: does it matter? J Health Soc Behav 1995;36:1-10.

27. Tong A, Sainsbury P, Craig J. Consolidated criteria for reporting qualitative research (COREQ): a 32-item checklist for interviews and focus groups. Int J Qual Health Care 2007;19:349-57.

28. Andersen RM, Davidson PL. Improving access to care in America: individual and contextual indicators. Changing the US health care system: key issues in health services policy and management. San Francisco, CA: Jossey-Bass, 2007:3-31.

29. Makowski D, Sperduti M, Blanchet S, et al. [Emotion regulation and the cognitive decline in aging: beyond the paradox]. Geriatr Psychol Neuropsychiatr Vieil 2015;13:301-8.

30. Body R, Kaide E, Kendal S. Not all suffering is pain: sources of patients' suffering in the emergency department call for improvements in communication from practitioners. Emerg Med $J$ 2015;32:15-20.

31. Markus HR, Kitayama S. Culture and the self: implications for cognition, emotion, and motivation. Psychol Rev 1991;98:224.

32. Russell JA. Culture and the categorization of emotions. Psychol Bull 1991;110:426 